\title{
Comparing methods for assessing bronchial responsiveness in children: single step cold air challenge, multiple step cold air challenge, and histamine provocation
}

\author{
M. Modl, E. Eber, B. Steinbrugger, E. Weinhandl, M.S. Zach
}

Comparing methods for assessing bronchial responsiveness in children: single step cold air challenge, multiple step cold air challenge, and histamine provocation. M. Modl, E. Eber, B. Steinbrugger, E. Weinhandl, M.S. Zach. CERS Journals Ltd 1995.

ABSTRACT: Cold air challenge $(\mathrm{CACh})$ can be applied by either a single step (SSCACh) or a multiple step (MSCACh) protocol. The interrelationship of the responses of the different protocols has not yet been studied. Furthermore, there is contradictory information on the correlation of cold air challenge responses to the outcome of pharmacological provocations.

A single and a multiple step cold air challenge and a histamine provocation were performed in random order on three consecutive days on 28 children and adolescents with bronchial asthma, who were currently symptom- and medication-free. Single step cold air challenge consisted of a 4 min isocapnic hyperventilation of dry, $-10^{\circ} \mathrm{C}$ air; the subject's response was quantified by the induced change in forced expiratory volume in one second (FEV1). Multiple step cold air challenge consisted of a series of $3 \mathrm{~min}$, cold dry air hyperventilation steps from 20 to $80 \%$ of maximal voluntary ventilation (MVV); response was expressed as the provocative dose causing a 10\% fall in FEV1 (PD10). Histamine provocation consisted of a series of 2 min inhalations of stepwise increasing histamine concentrations from 0.03 to 8.0 $\mathrm{mg} \cdot \mathrm{mL}^{-1}$; response was expressed as the provocative concentration of histamine causing a $20 \%$ fall in FEV1 (PC20).

Change in FEV1 ( $\triangle$ FEV1) (SSCACh) correlated closely with PD10 (MSCACh); scatter around the regression line was minimal. With one exception, both types of CACh identified the same subjects as hyper- and normoresponsive. $\triangle F E V 1$ (SSCACh) correlated significantly to $\mathrm{PC} 20$ (histamine), but scatter around the regression line was substantial. The correlation of PD10 (MSCACh) to PC20 (histamine) failed to reach statistical significance.

These results indicate that the stimulus applied and the bronchoconstrictor mechanism activated, and not the challenge protocol, determine the outcome of a cold air challenge. In clinical practice, a brief single step cold air challenge can substitute for a more time-consuming multiple step cold air challenge. As nonpharmacological challenges seem to measure a different type of bronchial responsiveness, neither a single step nor a multiple step cold air challenge can substitute for a pharmacological provocation.

Eur Respir J., 1995, 8, 1742-1747.
Respiratory and Allergic Disease Division, Paediatric Department, University of Graz, Graz, Austria.

\section{Correspondence: M.S. Zach}

Respiratory and Allergic Disease Division Paediatric Department

University of Graz

Univ.-Kinderklinik

Auenbruggerplatz 30

A-8036 Graz

Austria

Keywords: Bronchial responsiveness cold dry air challenge histamine provocation

Received: February 31995

Accepted after revision May 201995
Bronchial responsiveness is measured either by inhaling pharmacologically active substances, such as histamine or methacholine, or by applying physical stimuli like the hyperventilation of cold, dry air.

Cold air challenge (CACh) can be performed in two ways. Several paediatric groups have advocated a single step approach, i.e. single step cold air challenge (SSCACh); after applying one dose of cold dry air hyperventilation, the subject's response is quantified in terms of lung function changes effected [1-5]. Other authors have developed a multiple step approach with progressively increasing levels of cold dry air hyperventilation, i.e. multiple step cold air challenge (MSCACh); in analogy to pharmacological provocations, a dose-response curve is constructed and bronchial responsiveness is expressed as that dimension of hyperventilation that causes a certain predetermined change of lung function $[6,7]$.

The relationships between responses to $\mathrm{CACh}$ and those to pharmacological challenges have been studied repeatedly. Those studies that compared a MSCACh with a histamine or methacholine provocation demonstrated relatively close correlations [8-12]. Several comparisons between pharmacological challenges and a SSCACh, however, have produced contradictory results. One group found close correlations [13], whilst others observed only 
weak ones or none at all $[3,14,15]$. A recent comparison between responses to a SSCACh and those to a histamine provocation in 123 paediatric asthma patients resulted in a correlation that was statistically significant, but showed a wide scatter of the data points around the regression line [16].

These findings raise important questions. The obvious difference in stimuli applied and mechanisms activated between $\mathrm{CACh}$ and pharmacological challenges presents a probable explanation for the weakness of such SSCACh-histamine response correlations. However, the fact that the comparisons quoted above of a MSCACh with pharmacological provocations found relatively close correlations suggests an alternative explanation. It might just be the difference in challenge procedures, i.e. multiple step versus single step protocol, that is responsible for such a weak SSCACh-histamine response correlation. This question of different stimuli versus different challenge procedures could be clarified by directly comparing responses to a SSCACh with those to a MSCACh, but paradoxically, such directly comparative studies had not been performed.

In the present study, we therefore measured the responses of 28 paediatric asthma patients to a SSCACh, a MSCACh, and a histamine provocation, in order to answer the following questions: 1) Is it rather the difference in stimuli or the difference in challenge procedures that accounts for weak SSCACh-histamine response correlations? 2) Can a brief and simple SSCACh substitute for a more complex and time-consuming MSCACh? and 3) Can a CACh, either as SSCACh or MSCACh, substitute for measuring bronchial responsiveness by a pharmacological challenge?

\section{Methods}

\section{Subjects}

Subjects were participants of an ongoing epidemiological study that evaluates all asthma patients of this paediatric respiratory centre $1.5 \mathrm{yrs}$ after having become symptom-free, and $1 \mathrm{yr}$ after having become medicationfree. This epidemiological study aims at determining the prognostic value of lung functions, bronchial responsiveness, and allergy skin test reactivity by performing these measurements and then following the patients clinically and with repeat measurements. This group of subjects was felt to be ideally suited for a comparison of different bronchial provocation methods for two reasons: 1) two previous studies had shown this group to have a wide range of bronchial responsiveness [16, 17]; and 2) the absence of any anti-asthma medication that could influence different challenge mechanisms differently.

Initially, 30 patients were recruited for the present study. Two subjects were excluded; one refused to participate in more than two challenges, and one remained unable to hyperventilate accurately in the SSCACh and MSCACh. The remaining 28 subjects (17 boys and 11 girls) had a mean age of $15 \mathrm{yrs}(\mathrm{SD}=3 \mathrm{yrs}$, range $10-20$ yrs). All were well trained to cooperate with lung function testing and bronchial provocation procedures. All were long-term patients of this paediatric asthma centre, who, before becoming symptom-free, had met the clinical definition of bronchial asthma [18], and had been treated with various anti-asthma medications for a minimum of 3 yrs before becoming medication-free. Twenty five of them showed one or more positive skin test reaction(s) to common environmental allergens.

\section{SSCACh}

SSCACh was performed according to an established protocol $[3,5]$. Cold, dry air was produced by a commercially available heat exchanger (RHES, Jaeger, Wuerzburg, Germany). After the measurement of prechallenge FEV1, subjects hyperventilated absolutely dry, $-10^{\circ} \mathrm{C}$ air at $75 \%$ of their maximal voluntary ventilation (MVV) for $4 \mathrm{~min}$. The correct level of hyperventilation was maintained by having the subjects compete with a target balloon. $\mathrm{A} \mathrm{CO}_{2}$-analyser continuously monitored the $\mathrm{CO}_{2}$ concentration in the expired air, and $\mathrm{CO}_{2}$ was added to the inspired air in order to keep the subject eucapnic. Three minutes after termination of the SSCACh, FEV1 was measured again. The change in FEV1 $(\triangle \mathrm{FEV} 1)$ from the pre- to the postchallenge measurement was expressed in percentage baseline FEV1.

For this method, a $\triangle \mathrm{FEV} 1$ of minus $9 \%$ or more defines airway hyperreactivity $[3,19,20]$.

\section{MSCACh}

MSCACh was performed in accordance with a previous standardization [6]. Cold, dry air was provided by the same heat exchanger that was used for the SSCACh. Each step of this MSCACh protocol consisted of hyperventilating absolutely dry, $-10^{\circ} \mathrm{C}$ air for $3 \mathrm{~min}$. The first hyperventilation was carried out with $10 \%$ of the subject's MVV; this was followed by stepwise increasing the level of hyperventilation to 20,40, 60 and $80 \%$ MVV. The FEV1 was measured before, and at 30, 90 and $180 \mathrm{~s}$ after each hyperventilation period. The challenge was stopped when the FEV1 had fallen by $20 \%$ or more from baseline, or after having gone through all steps of hyperventilation. A dose-response curve was constructed by plotting the percentage fall in FEV1 against the hyperventilation dose on a log scale. The result of this MSCACh was expressed as the provocative dose of hyperventilation causing a $10 \%$ fall in FEV1 (PD10).

For this method, a PD10 of $80 \%$ MVV or below defines airway hyperreactivity [6, 7].

\section{Histamine provocation}

The histamine provocation was performed according to the present Austrian standardization [21], which is based on the method of COCKCROFT et al. [22]. As a tidal breathing method, this protocol is identical with a later European standardization [7], with the exception of using a nebulizer with a higher output. 
Briefly, each step of this multiple step protocol consisted of a 2 min inhalation by quiet tidal breathing through a mouthpiece using a noseclip. The first aerosol, inhaled after the prechallenge measurement of FEV1, was the diluent, and this was followed at $5 \mathrm{~min}$ intervals by doubling concentrations of histamine from 0.03 to $8.0 \mathrm{mg} \cdot \mathrm{mL}^{-1}$. The FEV1 was measured before, and at 30 and $90 \mathrm{~s}$ after each inhalation. The test was stopped when the FEV1 had fallen by $20 \%$ or more, or after having gone through all concentrations of histamine. A dose-response curve was constructed by plotting the percentage fall in FEV1 against the concentration of histamine on a log scale. The result was expressed as the provocative concentration of histamine causing a $20 \%$ fall in $\mathrm{FEV}_{1}\left(\mathrm{PC}_{20}\right)$.

Based on the output of the nebulizer used (Inhalierboy, Pari, Starnberg, Germany), a PC20 under $4 \mathrm{mg} \cdot \mathrm{mL}^{-1}$ defines airway hyperreactivity [21].

\section{Study protocol}

The three challenges were performed in random order, at 10 a.m., on three consecutive days. This $24 \mathrm{~h}$ interval was felt to be long enough to avoid refractoriness, and short enough to minimize spontaneous intraindividual variation of bronchial responsiveness; short-term reproducibility of CACh-responses is high [3]. Before the first challenge, a brief history was obtained and a clinical examination was performed; patients presenting with a recent history and/or the symptoms of an acute respiratory infection or an allergen exposure were not accepted for testing but received a later (minimum interval 6 weeks) reappointment. Before each challenge, subjects rested for $1 \mathrm{~h}$ in a controlled climate $\left(20^{\circ} \mathrm{C}, 40 \%\right.$ relative humidity).

Lung function testing was performed with a waterfilled spirometer (Spiro-Junior, Jaeger, Wuerzburg, Germany) in accordance with standardized guidelines [23]. FEV1 was expressed in absolute terms and as percentage predicted based on established reference standards [24]. After each challenge, recovery of lung function was observed for $20 \mathrm{~min}$; if by then the FEV1 had not returned to baseline, the patient was treated with nebulized salbutamol. Such treatment was also administered immediately after the challenge, when the patient subjectively felt any shortness of breath.

Informed consent to the study was obtained from patients and parents. The study was approved by the Ethics Committee of the local medical faculty.

The least squares correlation analysis was used for the statistical comparison of the responses to the three different challenges. Prechallenge lung function was compared by one-way analysis of variance. A p-value of 0.05 and below was taken as indicating statistical significance.

\section{Results}

Baseline FEV1 measurements before SSCACh (95.9 \pm $11.0 \%$ predicted), MSCACh $(95.0 \pm 10.8 \%$ pred $)$, and the histamine provocation $(96.0 \pm 13.5 \%$ pred $)$ did not differ significantly.

$\triangle \mathrm{FEV} 1$ in the SSCACh ranged from 0 to $-54 \%$ baseline. In the MSCACh, 13 subjects did not bronchoconstrict sufficiently for measuring a PD10. In the remaining 15 subjects, PD10 ranged from 18 to $80 \% \mathrm{MVV}$. In the histamine provocation, a $20 \%$ fall of FEV1 could not be effected in three subjects. In the remaining 25 subjects, PC20 ranged from 0.2 to $7.0 \mathrm{mg} \cdot \mathrm{mL}^{-1}$ histamine.

The correlation of $\triangle \mathrm{FEV}_{1}$ (SSCACh) with PD10 (MSCACh) is shown in figure 1 . The 13 subjects without a measured PD10 (MSCACh) had to be excluded from analysis. For the remaining 15 subjects, this correlation was highly significant, with only a minimal scatter of the data points around the regression line. Thirteen subjects were defined as normoresponsive, and 14 as hyperresponsive, both by SSCACh and MSCACh criteria. One subject with a PD10 (MSCACh) of 68\% MVV narrowly missed the SSCACh definition of hyperresponsiveness with a $\triangle \mathrm{FEV} 1$ of $-8 \%$.

The correlation of $\triangle \mathrm{FEV} 1$ (SSCACh) with PC20 (histamine) is shown in figure 2. The three subjects without a

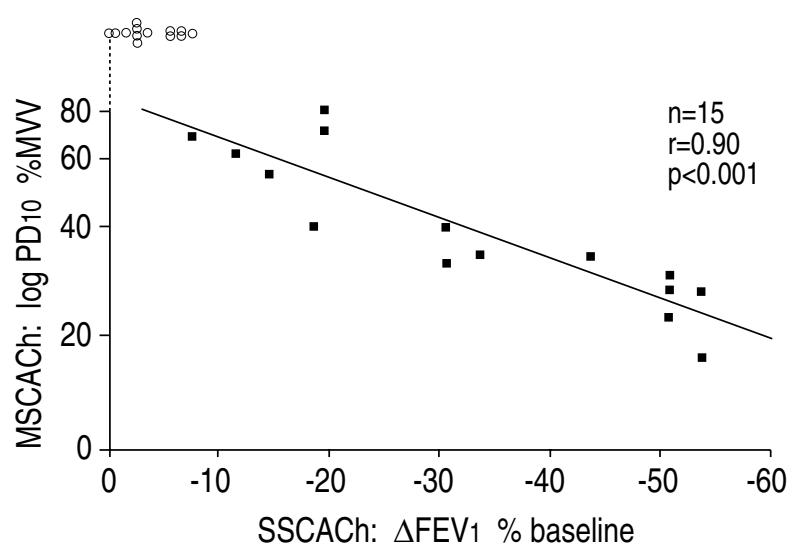

Fig. 1. - Correlation of $\triangle \mathrm{FEV}_{1}$ (SSCACh) with PD10 (MSCACh). Open symbols characterize the $\triangle F^{\prime} V_{1}$ (SSCACh) of the 13 subjects without a measured PD10 (MSCACh) that were excluded from correlation analysis. $\triangle \mathrm{FEV} 1$ : difference in forced expiratory volume in one second; SSCACh: single step cold air challenge; MSCACh: multiple step cold air challenge; PD10: provocative dose causing a $10 \%$ fall in FEV1; MVV: maximal voluntary ventilation.

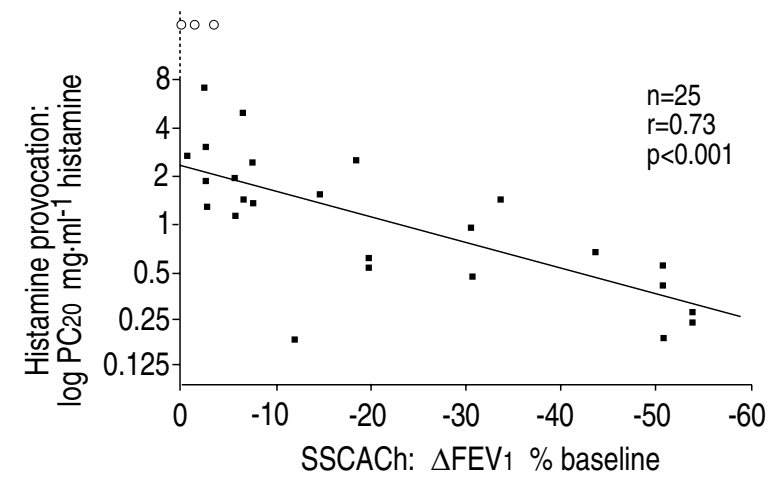

Fig. 2. - Correlation of $\triangle \mathrm{FEV}_{1}$ (SSCACh) with $\mathrm{PC}_{20}$ (histamine). Open symbols characterize the $\triangle \mathrm{FEV}_{1}$ (SSCACh) of the three subjects without a measured $\mathrm{PC}_{20}$ (histamine) that were excluded from correlation analysis: $\mathrm{PC} 20$ : provocative concentration of histamine causing a $20 \%$ fall in forced expiratory volume in one second. For further abbreviations see legend to figure 1 . 


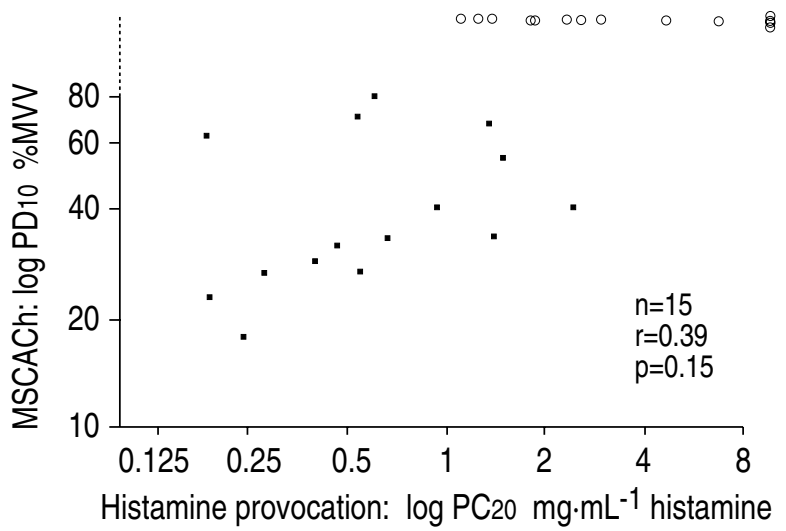

Fig. 3. - Correlation of PD10 (MSCACh) with PC20 (histamine). Open symbols characterize the PC20 (histamine) of the 10 subjects without a measured PD10 (MSCACh) that were excluded from correlation analysis. In addition, there are three open symbols for those subjects with neither a measured PD10 (MSCACh) nor a measured PC20 (histamine). For abbreviations see legends to figures 1 and 2.

measured PC20 (histamine) had to be excluded from analysis. For the remaining 25 subjects, this correlation was statistically significant. As evident from the figure, this correlation was markedly improved by a group of SSCACh-normoresponsive individuals with a $\triangle \mathrm{FEV} 1$ under $-9 \%$ baseline. For some of the residual data points, scatter around the regression line was considerable. When the correlation analysis was restricted to the subgroup of SSCACh-hyperresponsive individuals, $r$ decreased to 0.45 and statistical significance was lost. Five subjects were defined as normoresponsive, and 14 as hyperresponsive, both by SSCACh and histamine criteria. The remaining nine were found to be hyperresponsive in the histamine provocation, but remained normoresponsive in the SSCACh.

The correlation of PD10 (MSCACh) and PC20 (histamine) is shown in figure 3 . The 13 subjects without a measured PD10 (MSCACh), including those three without a measured PC20 (histamine), had to be excluded from analysis. For the remaining 15 subjects, this correlation did not reach statistical significance. Five subjects were defined as normoresponsive, and 15 as hyperresponsive, both by MSCACh and histamine criteria. The remaining eight were found to be hyperresponsive in the histamine provocation, but remained normoresponsive in the MSCACh.

\section{Discussion}

This study demonstrates a close SSCACh-MSCACh response correlation, and a weaker relationship between the outcome of these challenges and the results of a routine histamine provocation. Thus, the questions asked initially can be answered as follows: 1) It is the difference in applied stimuli and activated mechanisms, and not the difference in challenge procedures, that accounts for the weak SSCACh-histamine response correlations observed previously; 2) for practical purposes, a simple and less time-consuming SSCACh can substitute for a
MSCACh; and 3) as nonpharmacological challenges seem to measure a different type of bronchial responsiveness, neither a SSCACh nor a MSCACh can substitute for a histamine provocation.

In the case of the SSCACh, a size-corrected, predetermined bronchoconstrictor dose was administered, and bronchial responsiveness was quantified in terms of the lung function changes effected. In the case of the MSCACh, a dose-response curve was constructed and bronchial responsiveness was characterized by that hyperventilation dose that effected a certain predetermined change of lung function. In spite of these procedural differences, induced bronchial responses to the SSCACh, and to the MSCACh were found to be closely correlated. With one exception, both types of CACh defined the same subjects as normo- and hyperresponsive. This finding illustrates that it is the applied stimulus and the activated bronchoconstrictor mechanism, and not the protocol of the challenge, that determines the outcome of a CACh.

The findings of the present study have practical implications. Some paediatric groups have standardized a relatively simple SSCACh [1-5], whilst other authors have advocated a more complex multiple step approach [6]. The present study, as the first directly comparative evaluation of these two techniques, demonstrates that there is little reason for going through a time-consuming MSCACh protocol. From a paediatric viewpoint, this is an important finding, as a brief SSCACh can be applied more easily to paediatric patients with limited compliance. One additional advantage of the SSCACh is that it quantifies bronchial responsiveness in all subjects that undergo the challenge. In contrast, CACh-normoresponsive subjects will not bronchoconstrict sufficiently in the MSCACh to define a PD10; as illustrated by the present study, one is then left without a quantified response. This is especially disadvantageous when doing epidemiological work, which involves not only subjects with clearly increased bronchial responsiveness. The whole bronchial response spectrum of a given population can never be characterized by challenges, that do not quantify the response of each individual subject.

One theoretical reason for preferring a MSCACh to a SSCACh could be the relative safety of a protocol that increases the bronchoconstrictor dose gradually in closely monitored steps [6]. Practical experience shows, however, that the SSCACh is also a very safe challenge procedure. From 1982 to the end of 1994, 1,872 SSCAChs were performed in this paediatric respiratory centre; in spite of the fact that $78 \%$ of these challenges were performed in children with the established diagnosis of bronchial asthma, not one single serious complication occurred. This relative safety of the SSCACh might stem from reaching a maximum reaction plateau not only in normo- but also in hyperresponsive individuals [5]. In addition, SSCACh does not produce late asthmatic reactions [25].

In contrast to closely correlated SSCACh and MSCACh responses, histamine measurements on the one side and the outcome of both types of $\mathrm{CACh}$ on the other were 
found to be less closely correlated. Whilst the SSCAChhistamine response correlation reached statistical significance, the MSCACh-histamine response correlation failed to do so. On first sight, these results seem to contradict each other. How can the closely correlated responses to two challenges show marked differences in their correlation to the result of a third one? A comparison of figure 2 and 3 offers a probable answer to this question. Thirteen subjects without a measured PD10 (MSCACh) had to be excluded from the MSCAChhistamine response correlation. In contrast, the SSCAChhistamine response correlation only lacked three subjects without a measured PC20 (histamine). Thus, the statistical analysis of the SSCACh-histamine response correlation did benefit from a group of data points from SSCACh-normoresponsive individuals, who, with one exception, did not participate in the statistical analysis of the MSCACh-histamine response correlation.

The observed failure of the MSCACh-histamine response correlation to reach statistical significance is in marked contrast to the results of previous studies that compared the responses to a MSCACh with those to pharmacological provocations, and found statistically significant correlations [8-12]. This discrepancy cannot be explained by histamine-methacholine response differences, as these two types of pharmacological provocation produce closely correlated bronchial reactions [26, 27]. One obvious difference is that previous studies were performed in adults, whilst the present one employed children and adolescents. Applying challenges to children with respiratory tracts of widely differing dimensions makes size correction of the bronchoconstrictor stimulus a crucial issue. Such size correction is relatively simple for the voluntary hyperventilation of cold, dry air of the dimension, which is adjusted according to the subject's own prechallenge MVV. Aerosol application of bronchoconstrictors, however, faces major unresolved problems of size correction, such as dilution by air entrainment and widely varying deposition patterns [28]. Lack of effective size correction of the aerosol stimulus in the histamine provocation could, thus, have contributed to the presently observed weak MSCAChhistamine response correlation. Such problems of size correction, however, might have had a much smaller impact on previous studies in adults, which, as a consequence, were able to document closer response correlations. If this speculation is valid, one has to conclude that a physical stimulus, such as $\mathrm{CACh}$, is much better suited for comparing bronchial responses in children over a wider size and age range, than any provocation that applies aerosolized bronchoconstrictors.

Both types of CACh produced closely correlated responses. The SSCACh-histamine response correlation, however, showed considerable scatter, and the MSCAChhistamine response correlation failed to reach statistical significance altogether. In addition to the problems discussed above with size-correction of the aerosol stimulus, this finding might stem from the obvious differences in stimuli applied and mechanisms activated, that exist between a CACh and a histamine provocation. Unlike aerosols of histamine and methacholine, CACh does not act directly on bronchial smooth muscle, but instead invokes intermediate events, such as the release of bronchoactive mediators from cells in the airway lumen and submucosa [29]. When different challenges apply different stimuli and activate different bronchoconstrictor mechanisms, however, they should be measuring different types of airway responsiveness. The practical conclusion is that CACh cannot substitute for a pharmacological provocation, such as histamine, in the measurement of bronchial responsiveness, and vice versa.

This difference between responses to pharmacological and nonpharmacological challenges is also illustrated by the histamine provocation defining more subjects as hyperreactive than the $\mathrm{CACh}$. This finding confirms the result of a previous study, that compared a SSCACh with a histamine provocation [16]. It is also in agreement with the work of GALDES-SEBALDT et al. [30], who compared nonpharmacological and pharmacological challenges for identifying bronchial asthma in a paediatric population; they found that a CACh is less sensitive but more specific for defining asthma than a conventional methacholine challenge. Such findings again indicate that, depending on the choice of bronchoconstrictor stimulus, one is looking at two somehow related but nevertheless different types of bronchial responsiveness.

In conclusion, this study found that SSCACh and MSCACh, employing the same stimulus but following different protocols, produce closely correlated responses. This finding indicates that, for practical purposes, a SSCACh can substitute for a more complex MSCACh. Responses to both types of CACh were found to be less closely related to the outcome of a standardized histamine provocation. This finding suggests problems with the size correction of an aerosol bronchoconstrictor stimulus in a paediatric population. In addition, it indicates that responses to any type of challenge remain, at least in part, specific for the stimulus applied.

\section{References}

1. McLaughlin FJ, Dozor AJ. Cold air inhalation challenge in the diagnosis of asthma in children. Pediatrics 1983; 72: 503-509.

2. Tal A, Pasterkamp H, Serrette C, Leahy F, Chernick V. Response to cold air hyperventilation in normal and in asthmatic children. J Pediatr 1984; 104: 516-521.

3. Zach M, Polgar G, Kump H, Kroisel P. Cold air challenge of airway hyperreactivity in children: practical application and theoretical aspects. Pediatr Res 1984; 18: 469-478.

4. Reisman J, Mappa L, deBenedictis F, McLaughlin J, Levison H. Cold air challenge in children with asthma. Pediatr Pulmonol 1987; 3: 251-254.

5. Zach MS, Polgar G. Cold air challenge of airway hyperreactivity in children: dose-response interrelation with a reaction plateau. J Allergy Clin Immunol 1987; 80: 9-17.

6. Assoufi BK, Dally MB, Newman-Taylor AJ, Denisen DM. Cold air test: a simplified standard method for airway reactivity. Bull Eur Physiopathol Respir 1986; 22: 349-357. 
7. Sterk PJ, Fabbri LM, Quanjer PH, et al. Airway responsiveness.: standardized challenge testing with pharmacological, physical and sensitizing stimuli in adults. Eur Respir J 1993; 6 (Suppl. 16): 53-83.

8. Hargreave FE, Ryan G, Thomson NC, et al. Bronchial responsiveness to histamine or methacholine in asthma: measurement and clinical significance. J Allergy Clin Immunol 1981; 68: 347-355.

9. O'Byrne PM, Ryan G, Morris M, et al. Asthma induced by cold air and its relationship to nonspecific bronchial responsiveness to methacholine. Am Rev Respir Dis 1982; 125: 281-285.

10. Weiss JW, Rossing TH, McFadden ER, Ingram RH. Relationship between bronchial responsiveness to hyperventilation with cold air and methacholine in asthma. $J$ Allergy Clin Immunol 1983; 72: 140-144.

11. Heaton RW, Henderson AF, Costello JF. Cold air as a bronchial provocation technique: reproducibility and comparison with histamine and methacholine inhalation. Chest 1984; 86: 810-814.

12. Tessier P, Ghezzo H, L'Archeveque J, Cartier A, Malo JL. Shape of the dose-response curve to cold air inhalation in normal and asthmatic subjects. Am Rev Respir Dis 1987; 136: 1418-1423.

13. Aquilina AT. Comparison of airway reactivity induced by histamine, methacholine, and isocapnic hyperventilation in normal and asthmatic subjects. Thorax 1983; 38: 766-770.

14. Nair N, Hopp RJ, Alper BJ, Bewtra AK, Townley RG. Correlation of methacholine-induced nonspecific bronchial reactivity and cold air hyperventilation challenge. Ann Allergy 1986; 56: 226-228.

15. Filuk RB, Serrette C, Anthonisen NR. Comparison of responses to methacholine and cold air in patients suspected of having asthma. Chest 1989; 95: 948-952.

16. Steinbrugger B, Eber E, Modl M, Weinhandl E, Zach MS. A comparison of a single-step cold-dry air challenge and a routine histamine provocation for the assessment of bronchial responsiveness in children and adolescents. Chest (in press).

17. Steinbrugger B, Zach M. Bronchiale Reaktivität bei klinisch beschwerdefreien jugendlichen Asthmapatienten. Monatsschr Kinderheilkd 1990; 138: 389-391.

18. National Heart, Lung and Blood Institute, National Institutes of Health, Bethesda, Maryland. International consensus report on diagnosis and treatment of asthma. Eur Respir J 1992; 5: 601-641.

19. Zach M, Hadl M. Die Kaltlufthyperventilationsprovokation-
Normalwertbereiche einzelner Lungenfunktionsparameter im Kindesalter. Atemw Lungenkrkh 1987; 7: 298-299.

20. Nicolai T, Mutius EV, Reitmeir P, Wjst M. Reactivity to cold-air hyperventilation in normal and in asthmatic children in a survey of 5,697 schoolchildren in southern Bavaria. Am Rev Respir Dis 1993; 147: 565-572.

21. Österreichische Gesellschaft für Lungenerkrankungen und Tuberkulose-Arbeitsgemeinschaft für klinische Atemphysiologie. Empfehlungen zur Standardisierung der inhalativen Provokation zur Messung der unspezifischen bronchialen Reaktivität. Prax Klin Pneumol 1986; 40: 356-364.

22. Cockcroft DW, Killian DN, Mellon JJA, Hargreave FE. Bronchial reactivity to inhaled histamine: a method and clinical survey. Clin Allergy 1977; 7: 235-243.

23. Quanjer PH, Tammeling GJ, Cotes JE, Pedersen OF, Peslin R, Yernault JC. Lung volumes and forced ventilatory flows. Report working party standardization of lung function tests, European Coal and Steel Community. Official statement of the European Respiratory Society. Eur Respir J 1993; 6 (Suppl. 16): 5-40.

24. Hibbert ME, Lannigan A, Landau LJ, Phelan PD. Lung function values from a longitudinal study of healthy children and adolescents. Pediatr Pulmonol 1989; 7: 101109.

25. Varga EM, Eber E, Zach MS. Cold air challenge for measuring airway reactivity in children: lack of a late asthmatic reaction. Lung 1990; 168: 267-272.

26. Juniper EF, Frith PA, Dunnett C, Cockcroft DW, Hargreave FE. Reproducibility and comparison of responses to inhaled histamine and methacholine. Thorax 1978; 33: 705-710.

27. Juniper EF, Frith PA, Hargreave FE. Airway responsiveness to histamine and methacholine: relationship to minimum treatment to control symptoms of asthma. Thorax 1981; 36: 575-579.

28. LeSouef PN. Can measurements of airway responsiveness be standardized in children? Eur Respir J 1993: 6: 1085-1087.

29. Pliss LB, Ingenito EP, Ingram RH, Pichurko B. Assessment of bronchoalveolar cell and mediator response to isocapnic hyperpnea in asthma. Am Rev Respir Dis 1990; 142: 73-78.

30. Galdes-Sebaldt M, McLaughlin FJ, Levison H. Comparison of cold air, ultrasonic mist, and methacholine inhalations as tests of bronchial reactivity in normal and asthmatic children. J Pediatr 1985; 107: 526-530. 